\title{
ヘь
}

The Axiom of Determinancy Implies Dependent Choices in L(R)

Author(s): Alexander S. Kechris

Source: The Journal of Symbolic Logic, Vol. 49, No. 1 (Mar., 1984), pp. 161-173

Published by: Association for Symbolic Logic

Stable URL: http://www.jstor.org/stable/2274099

Accessed: 20/05/2013 16:25

Your use of the JSTOR archive indicates your acceptance of the Terms \& Conditions of Use, available at http://www.jstor.org/page/info/about/policies/terms.jsp

JSTOR is a not-for-profit service that helps scholars, researchers, and students discover, use, and build upon a wide range of content in a trusted digital archive. We use information technology and tools to increase productivity and facilitate new forms of scholarship. For more information about JSTOR, please contact support@jstor.org. 


\title{
THE AXIOM OF DETERMINACY IMPLIES DEPENDENT CHOICES IN $L(\mathbf{R})$
}

\author{
ALEXANDER S. KECHRIS ${ }^{1}$
}

\begin{abstract}
We prove the following Main Theorem: $\mathrm{ZF}+\mathrm{AD}+V=L(\mathrm{R}) \Rightarrow \mathrm{DC}$. As a corollary we have that $\operatorname{Con}(\mathrm{ZF}+\mathrm{AD}) \Rightarrow \operatorname{Con}(\mathrm{ZF}+\mathrm{AD}+\mathrm{DC})$. Combined with the result of Woodin that $\operatorname{Con}(\mathrm{ZF}+\mathrm{AD}) \Rightarrow \mathrm{Con}\left(\mathrm{ZF}+\mathrm{AD}+\neg \mathrm{AC}^{\omega}\right)$ it follows that $\mathrm{DC}$ (as well as $A C^{\omega}$ ) is independent relative to $\mathrm{ZF}+\mathrm{AD}$. It is finally shown (jointly with $\mathrm{H}$. Woodin) that $\mathrm{ZF}+\mathrm{AD}+\neg \mathrm{DC}_{\mathbf{R}}$, where $\mathrm{DC}_{\mathbf{R}}$ is $\mathrm{DC}$ restricted to reals, implies the consistency of $\mathrm{ZF}+\mathrm{AD}+\mathrm{DC}$, in fact implies $\mathbf{R}^{\#}$ (i.e. the sharp of $L(\mathbf{R})$ ) exists.
\end{abstract}

§1. Introduction. 1.1. For each set $A \subseteq \mathbf{R}\left(=\omega^{\omega}\right.$, the set of "reals") consider the associated infinite game $G_{A}$ :

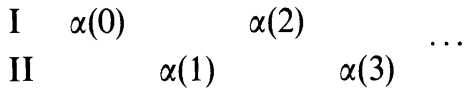

in which player I wins if $\alpha \in A$, and II wins otherwise. We call $A$ determined if the corresponding game $G_{A}$ is determined, i.e. either player I or player II has a winning strategy. The Axiom of Determinacy (AD) is the statement: $\forall A \subseteq \mathbf{R}(A$ is determined). Let also DC be the Axiom of Dependent Choices: For every nonempty set $X$ and every relation $R$ on $X$,

$$
\forall a \in X \exists b \in X R(a, b) \Rightarrow \exists f: \omega \rightarrow X \forall n R(f(n), f(n+1)) .
$$

Consider now the inner model $L(\mathbf{R})$ of all constructible from $\mathbf{R}$ sets, i.e. the smallest inner model of ZF containing the set of reals $\mathbf{R}$. It has been proposed as a strong hypothesis in set theory that $L(\mathbf{R})$ is a model of $\mathrm{AD}$ (for a discussion of these matters see [Mo2, 8I]), and an extensive theory of the structure of $L(\mathbf{R})$ has been developed over the years under this hypothesis. This theory requires at several key places the use of DC, which is the strongest form of choice compatible with AD that can hold in $L(\mathbf{R})$. It is of course easy to check, using the fact that DC holds in the universe, that $\mathrm{DC}$ is also true in $L(\mathbf{R})$. From the development of this theory until now, one gets the clear impression at this point that " $\mathrm{ZF}+\mathrm{DC}+\mathrm{AD}+V=L(\mathbf{R})$ " is a "complete" theory for $L(\mathbf{R})$, in the same sense as " $Z F+V=L$ " is a "complete" theory for the constructible universe $L$. There is one apparent difference however.

Received April 29, 1982.

${ }^{1}$ Research partially supported by NSF Grant No. MCS-8117804. The author is an A. P. Sloan Foundation Fellow. 
Although $\mathrm{ZF}+V=L$ implies the Axiom of Choice (AC), one seems to need to add to $\mathrm{ZF}+V=L(\mathbf{R})+\mathrm{AD}$ the choice principle $\mathrm{DC}$ needed in developing the theory of $L(\mathbf{R})$.

We show here that this is not necessary.

THEOREM. Assume $\mathrm{ZF}+\mathrm{AD}+V=L(\mathbf{R})$. Then $\mathrm{DC}$ holds.

Thus one has the full analogy.

$$
\frac{L}{\mathrm{ZF}+V=L} \sim \frac{L(\mathbf{R})}{\mathrm{ZF}+V=L(\mathbf{R})+\mathrm{AD}} .
$$

Although from the realistic point of view the foundational significance of this result is not immediately apparent (after all, DC holds in $L(\mathbf{R})$ ), it does demonstrate once again the power and elegance of the determinancy hypothesis. (It also helps save a lot of ink in the future.)

From the formal point of view we have also the following immediate corollary, which guarantees that the use of $\mathrm{DC}$ with $\mathrm{AD}$ is no more dangerous than $\mathrm{AD}$.

Corollary. Con $(\mathrm{ZF}+\mathrm{AD}) \Rightarrow \operatorname{Con}(\mathrm{ZF}+\mathrm{AD}+\mathrm{DC})$.

The question of the relative consistency of $\mathrm{DC}$ with $\mathrm{AD}$ has been raised during the early stages of the development of the theory of determinacy; see for instance [Ma, p. 687] or [Mo3, p. 32].

H. Woodin has proved the result corresponding to this corollary for $\neg \mathrm{DC}$; in fact, he shows:

THEOREM (Woodin). Con (ZF $+\mathrm{AD}) \Rightarrow \mathrm{Con}\left(\mathrm{ZF}+\mathrm{AD}+\neg \mathrm{AC}^{\omega}\right)$, where $\mathrm{AC}^{\omega}$ is the Countable Axiom of Choice, i.e. the statement that for all nonempty sets $X$ and all relations $R$ on $\omega \times X$, if $\forall n \exists a \in X R(n, a)$, then $\exists f: \omega \rightarrow X \forall n R(n, f(n))$. So we have the full independence of $\mathrm{DC}$ and $\mathrm{AC}^{\omega}$ from $\mathrm{ZF}+\mathrm{AD}$. We include Woodin's proof in $\S 3$ of the present paper with his permission.

This situation should be contrasted with that of $\mathrm{AD}_{\mathbf{R}}$ (the Axiom of Determinacy for games on $\mathbf{R}$ ), where Solovay [So] has shown that

$$
\mathrm{ZF}+\mathrm{AD}_{\mathbf{R}}+\mathrm{DC} \Rightarrow \mathrm{Con}\left(\mathrm{ZF}+\mathrm{AD}_{\mathbf{R}}\right) \text {. }
$$

1.2. Let us comment briefly now on some other results on the connection of $A D$ with choice principles and some open problems.

By $\mathrm{AC}_{\mathbf{R}}^{\omega}$ we mean the restriction of $\mathrm{AC}^{\omega}$ to the case of reals, i.e. where $X=\mathbf{R}$. Similarly let $\mathrm{DC}_{\mathbf{R}}$ be the restriction of $\mathrm{DC}$ to the case of reals, i.e. again where $X=\mathbf{R}$.

It has been noticed since the early days of determinacy (see $[\mathrm{My}]$ ) that

$$
\mathrm{ZF}+\mathrm{AD} \Rightarrow \mathrm{AC}_{\mathbf{R}}^{\omega} \text {. }
$$

(Consider the game where I plays $n, n_{1}, n_{2}, \ldots$, II plays $x(0), x(1), \ldots$, and II wins iff $R(n, x)$.) From this it follows easily that

$$
\mathrm{ZF}+\mathrm{AD}+V=L(\mathbf{R}) \Rightarrow \mathrm{AC}^{\omega},
$$

so that from $\mathrm{ZF}+\mathrm{AD}+V=L(\mathbf{R})$ one has at least the Countable Axiom of Choice Again to prove $\mathrm{DC}$ in $\mathrm{ZF}+\mathrm{AD}+V=L(\mathbf{R})$ it is enough to establish that

$$
\mathrm{ZF}+\mathrm{AD}+V=L(\mathbf{R}) \Rightarrow \mathrm{DC}_{\mathbf{R}} .
$$


This is what we prove in $\S 2$. Contrary to the case of $\mathrm{AC}_{\mathbf{R}}^{\omega}$, where a simple game gives immediately the choice principle, we do not know how to derive $\mathrm{DC}_{\mathbf{R}}$ directly by playing some sort of a game, which surely would be the most natural approach. Our proof is fairly roundabout and is based on the theory of scales in $L(\mathbf{R})$ developed recently by Martin, Moschovakis and Steel (see [MMS], and papers in [KMM]), and earlier work of Moschovakis on computing definability estimates for strategies (the Third Periodicity Theorem, [Mo2, 6E.1]). It would be desirable of course to have a more direct proof, which brings us to the following well-known question:

\section{Does $\mathrm{ZF}+\mathrm{AD}$ imply $\mathrm{DC}_{\mathbf{R}}$ ?}

Solovay [So] conjectures that it does not. Our theorem combined with Vopěnka's theorem (see [J, p. 293]) can be used to show that at least $\mathrm{ZF}+\mathrm{AD}+\neg \mathrm{DC}_{\mathbf{R}}$ is stronger than $\mathrm{ZF}+\mathrm{AD}$. This result is jointly due to $\mathrm{H}$. Woodin and the author.

THEOREM (WITH H. WoOdIN). $\mathrm{ZF}+\mathrm{AD}+\neg \mathrm{DC}_{\mathbf{R}} \Rightarrow \mathrm{Con}(\mathrm{ZF}+\mathrm{AD})$.

In the proof of this theorem one actually shows that $Z F+A D+\neg D_{\mathbf{R}}$ proves the existence of $\mathbf{R}^{\#}$ (i.e. the sharp of $L(\mathbf{R})$ ). So we can add to the above question the following:

If $\mathrm{ZF}+\mathrm{AD}+\neg \mathrm{DC}_{\mathbf{R}}$ is consistent, how strong is it? For instance, is it as strong as $\mathrm{ZF}+\mathrm{AD}_{\mathbf{R}}+\mathrm{DC}$ ?

1.3. We give in $\S 2$ the proof of the main theorem and in $\S 3$ the proof of the independence of $\mathrm{DC}$ from $\mathrm{ZF}+\mathrm{AD}$. In $\S 4$ we prove the result on the strength of $\mathrm{ZF}$ $+\mathrm{AD}+\neg \mathrm{DC}_{\mathbf{R}}$. Finally in $\S 5$ we discuss briefly the restriction of the present results to the context of projective sets, and the implications of PD (Projective Determinacy) to choice principles in analysis. We assume throughout that the reader is familiar with [Mo2] for general background in descriptive set theory.

§2. Proof of the main theorem. 2.1. Our proof is based on an elaboration of the Third Periodicity Theorem of Moschovakis [Mo2, 6E.1] and the recent work of Martin, Moschovakis and Steel (see [MMS] and articles in [KMM]) on scales in $L(\mathbf{R})$.

We will work for the remainder of $\S 2$ in $\mathrm{ZF}+\mathrm{AD}+V=L(\mathbf{R})$, and we will prove DC. As noted in the introduction we at least have $\mathrm{AC}^{\omega}$, the Countable Axiom of Choice.

Define first as usual the $J$-hierarchy for $L(\mathbf{R})$ (see $[\mathrm{St}])$ :

$$
J_{1}(\mathbf{R})=V_{\omega+1}
$$

$$
\begin{gathered}
J_{\alpha+1}(\mathbf{R})=\operatorname{rud}\left(J_{\alpha}(\mathbf{R})\right)=\text { the rudimentary closure of } J_{\alpha}(\mathbf{R}) \cup\left\{J_{\alpha}(\mathbf{R})\right\}, \text { for } \alpha>0 ; \\
\qquad J_{\lambda}(\mathbf{R})=\bigcup_{\alpha<\lambda} J_{\alpha}(\mathbf{R}) \text { for } \lambda \text { limit. }
\end{gathered}
$$

Then $L(\mathbf{R})=\bigcup_{\alpha \in \text { ord }} J_{\alpha}(\mathbf{R})$.

Since there is a definable (with no parameters) $\mathbf{F}:$ ORD $\times \mathbf{R} \stackrel{\text { onto }}{\longrightarrow} V$, it is easy to check that if $\mathrm{DC}$ fails then $\mathrm{DC}_{\mathbf{R}}$ fails, i.e. there is a relation $P \subseteq \mathbf{R} \times \mathbf{R}$ such that $\forall x \in \mathbf{R} \exists y \in \mathbf{R} P(x, y)$, but there is no $f: \omega \rightarrow \mathbf{R}$ with $\forall n R(f(n), f(n+1))$. Find a large enough ordinal $\xi$ such that $P$ belongs to $J_{\xi}(\mathbf{R})$, and then let $\alpha>1$ be the least ordinal $\leq \xi$ with $J_{\alpha}(\mathbf{R}){ }_{1}^{\mathbf{R}} J_{\xi}(\mathbf{R})$, where this notation means that every $\Sigma_{1}$ formula 
with parameters in $\mathbf{R} \cup\{\mathbf{R}\}$ which holds in $J_{\xi}(\mathbf{R})$, holds in $J_{\alpha}(\mathbf{R})$ as well. In particular

$$
J_{\alpha}(\mathbf{R}) \vDash \exists P \subseteq \mathbf{R}^{2}(\forall x \in \mathbf{R} \exists y \in \mathbf{R} P(x, y)) \wedge \neg \exists f: \omega \rightarrow \mathbf{R} \forall n P(f(n), f(n+1))
$$

(note that any $f: \omega \rightarrow \mathbf{R}$ "is" a real). So there is a counterexample $P$ to $\mathbf{D C}_{\mathbf{R}}$ (in the universe) which belongs in $J_{\alpha}(\mathbf{R})$. Suppose we could show that every relation $S \subseteq \mathbf{R} \times \mathbf{R}$ in $J_{\alpha}(\mathbf{R})$ can be uniformized (in the universe, not necessarily in $J_{\alpha}(\mathbf{R})$ ), i.e. there is $F: \mathbf{R} \rightarrow \mathbf{R}$ such that

$$
\exists y S(x, y) \Rightarrow S(x, F(x))
$$

Applying this to $P(x, y)$ find such an $F$, and let $f(0)=x_{0}$ (some fixed element of $\mathbf{R}$ ) and $f(n+1)=F(f(n))$. Then $f: \omega \rightarrow \mathbf{R}$ and $\forall n P(f(n), f(n+1))$, a contradiction.

So it is enough to prove this uniformization result for relations in $J_{\alpha}(\mathbf{R})$.

First note that there is a partial map

$$
\pi: \mathbf{R} \stackrel{\text { onto }}{\longrightarrow} J_{\alpha}(\mathbf{R})
$$

with $\Sigma_{1}\left(J_{\alpha}(\mathbf{R})\right)$ graph [here $\Sigma_{1}\left(J_{\alpha}(\mathbf{R})\right)$ means $\Sigma_{1}$ definable with only parameter $\mathbf{R}$ ]. This follows from a Skolem hull argument using the fact that there is no $1<\beta<\alpha$ with $J_{\beta}(\mathbf{R}) \prec_{1}^{\mathbf{R}} J_{\alpha}(\mathbf{R})$; see [St, 1.11]. In particular every set of reals in $J_{\alpha}(\mathbf{R})$ is $\Sigma_{1}$ definable in $J_{\alpha}(\mathbf{R})$ using only parameters from $\mathbf{R} \cup\{\mathbf{R}\}$. So it will be enough to show that if $A \subseteq \mathbf{R}$ is nonempty and $\Sigma_{1}$ definable in $J_{\alpha}(\mathbf{R})$ with parameters $r, \mathbf{R}(r$ a real), then it contains a member $x$ which is ordinal definable from $r$, $\mathbf{R}$. In fact to make things more concrete we will show that $x$ can be taken to be first-order definable from $r, \mathbf{R}$ in $J_{\alpha}(\mathbf{R})$.

To see how this implies the uniformization we need, let $S(x, y)$ be in $J_{\alpha}(\mathbf{R})$, say $\Sigma_{1}$ definable from $r, \mathbf{R}$. For each real $x$, let $F(x)=x$ if $\neg \exists y S(x, y)$. If $\exists y S(x, y)$, let $F(x)$ be the least real $z$ first-order definable from $\langle x, r\rangle, \mathbf{R}$ in $J_{\alpha}(\mathbf{R})$ such that $S(x, z)$, where "least" refers to some canonical enumeration of formulas.

2.2. Let us restate as a claim the basis result we want to prove.

Claim. Let $\alpha>1$ and let $A \subseteq \mathbf{R}$ be $\Sigma_{1}$ definable in $J_{\alpha}(\mathbf{R})$ with parameters $r, \mathbf{R}, r$ a real. If $A \neq \varnothing$ then $A$ contains a member $x$ which is first-order definable in $J_{\alpha}(\mathbf{R})$ with only parameters $r, \mathbf{R}$.

Our first impulse is to quote Theorem $2.1 \mathrm{in}$ [St], that asserts that if $\alpha$ is as above then the class of all sets of reals which are $\Sigma_{1}$ definable in $J_{\alpha}(\mathbf{R})$ with only parameter $\mathbf{R}$ has the scale property. That would surely do it, as there is a canonical ("leftmost branch") procedure for selecting an element from a set carrying scales (see [Mo2, 4E.3]). Unfortunately the proof of this theorem uses DC. However, all is not lost. By a careful analysis of Steel's proof and some appropriate modifications in it, we are lead to see that without DC we can still get a weaker version of scales, which we call quasiscales below. Then we simply observe that if we use the procedure of the Third Periodicity Theorem, instead of the standard leftmost branch procedure from scales, we can still canonically pick an element from a set carrying a quasiscale, and this completes our proof.

2.3. We give the details now.

Definition. Let $A \subseteq \mathbf{R}$. A quasiscale on $A$ is a sequence of relations $\leq_{0}, \leq_{1}, \leq_{2}, \ldots$, such that 
(a) Each $\leq_{i}$ is a linear preordering on $A$, i.e. Field $\left(\leq_{i}\right)=A$, and for $x, y, z \in A$

$$
x \leq_{i} x, x \leq_{i} y \vee y \leq_{i} x, x \leq_{i} y \wedge y \leq_{i} z \Rightarrow x \leq_{i} z .
$$

(b) Let $x<_{i} y \Leftrightarrow x \leq_{i} y \wedge y \not_{i} x$. Then for each $i$, there is no infinite descending chain for $<_{i}$.

(c) If $x_{0}, x_{1}, x_{2}, \ldots \in A$ and

(1) $x_{n} \rightarrow x$,

(2) for each $i$ there is $N_{i} \equiv N$ such that, for all $n \geq N, x_{n} \equiv_{i} x_{N}$ (i.e. $\left.x_{n} \leq_{i} x_{N} \wedge x_{N} \leq_{i} x_{n}\right)$

then

(3) $x \in A$, and

(4) for all $i, x \leq_{i} x_{N_{i}}$.

If (b) is replaced by:

(b*) For each $i$, every nonempty $X \subseteq A$ has an $\leq_{i}$-least element (i.e. $\leq_{i}$ is a prewellordering),

then we have the notion of a scale (see [Mo2]). Clearly, using $\mathrm{DC}_{\mathbf{R}}$ every quasiscale is a scale.

If a nonempty set $A$ carries a scale $\left\{\leq_{i}\right\}$ there is a canonical procedure for picking an element out of $A$ :

Let $A_{0}=\left\{x \in A: x\right.$ is least in $\left.\leq_{0}\right\}, A_{0}^{\prime}=\left\{x \in A_{0}: x(0)\right.$ is least $\}, A_{1}=\left\{x \in A_{0}^{\prime}: x\right.$ is least in $\left.\leq_{1}\right\}, A_{1}^{\prime}=\left\{x \in A_{1}: x(1)\right.$ is least $\}, \ldots$. Then $\bigcap_{n} A_{n}=\left\{x_{0}\right\}$ and $x_{0} \in A$.

This argument clearly does not necessarily work for a quasiscale. We shall see however that if instead of this procedure we use the idea of the Third Periodicity Theorem, we can still canonically pick an element from a nonempty set carrying a quasiscale. More precisely we can prove the following, where the definability estimate is grossly exaggerated. (Since it is sufficient for our purposes we will not try to state it in a sharp form).

Lemma. Suppose $A \subseteq \mathbf{R}$ carries a quasiscale $\left\{\leq_{i}\right\}$, and let $\Delta$ be a pointclass containing $A$ and the sequence $\left\{\leq_{i}\right\}$ (i.e. the relation $R(i, x, y) \Leftrightarrow\left(x, y \in A \wedge x \leq_{i} y\right)$ is in 4$)$, and closed under recursive substitutions, $\neg, \wedge, \vee$, and existential and universal quantification over $\mathbf{R}$. Then if $A$ is nonempty, $A$ contains a real (with graph) in $\Delta$.

Proof. First we turn the quasiscale $\left\{\leq_{i}\right\}$ on $A$ into a very good quasiscale $\left\{\leq_{i}^{*}\right\}$, i.e. one which has, beyond (a)-(c), also the following properties:

(d) If $x, y \in A$ then $x \leq_{n}^{*} y \Rightarrow \forall i \leq n\left(x \leq_{i}^{*} y\right)$

(e) If $x, y \in A$ and $x \equiv_{n}^{*} y$, then $x\lceil n+1=y\lceil n+1$.

The way we define $\left\{\leq_{i}^{*}\right\}$ from $\left\{\leq_{i}\right\}$ is by the usual lexicographical procedure as follows: For $x, y \in A$, put

$$
\begin{aligned}
x \leq_{i}^{*} y \Leftrightarrow x<_{0} y & \vee\left[x \equiv_{0} y \wedge x(0)<y(0)\right] \\
& \vee\left[x \equiv_{0} y \wedge x(0)=y(0) \wedge x<_{1} y\right] \\
& \vee \cdots \\
& \vee\left[x \equiv_{0} y \wedge x(0)=y(0) \wedge x \equiv_{1} y\right. \\
& \left.\wedge x(1)=y(1) \wedge \cdots \wedge x(i-1)=y(i-1) \wedge x<_{i} y\right] \\
& \vee\left[x \equiv_{0} y \wedge x(0)=y(0) \wedge \cdots \wedge x \equiv_{i} y \wedge x(i) \leq y(i)\right] .
\end{aligned}
$$


It is easy to verify that this is indeed a good quasiscale and moreover $\left\{\leq_{i}^{*}\right\}$ is also in $\Delta$. To simplify the notation we may therefore assume that the quasiscale $\left\{\leq_{i}\right\}$ is very good to start with.

Assume also below that $A \neq \varnothing$.

Motivated by the proof of the Third Periodicity Theorem, define for each $k \geq 0$ the following relation among all sequences $u$ from $\omega$ of length $k+1$ which are such that $\exists x \supseteq u(x \in A): v \leq^{k} u \Leftrightarrow$ Player II has a winning strategy in the following game:

$$
\begin{array}{lllll}
\text { I } & x(0) & & x(1) & \\
\text { II } & & y(0) & & y(1)
\end{array}
$$

let $x^{\prime}=u^{\cap} x$ and $y^{\prime}=v^{n} y$. Then II wins iff $\left[y^{\prime} \in A \wedge\left(x^{\prime} \notin A \vee y^{\prime} \leq_{k} x^{\prime}\right)\right]$. As in [Mo2, 6E.1] we can now verify that $\leq^{k}$ is a preordering on $W_{k}=\left\{u \in \omega^{k+1}: \exists x\right.$ $\supseteq u(x \in A)\}$ and moreover that there is no infinite descending $<^{k}$-chain. Since $W_{k}$ consists of finite sequences from $\omega$ this implies that actually $\leq^{k}$ is a prewellordering on $W_{k}$. Define then inductively, $u_{0} \subseteq u_{1} \subseteq \cdots \subseteq u_{k} \subseteq \cdots$, so that $u_{k} \in W^{k}$ and if $u_{k}=\left(a_{0}, \ldots, a_{k}\right)$ then for all $b$ with $\left(a_{0}, \ldots, a_{k-1}, b\right) \in W_{k}$ we have $\left(a_{0}, \ldots, a_{k}\right)$ $\leq^{k}\left(a_{0}, \ldots, a_{k-1}, b\right)$, and moreover $a_{k}$ is least with that minimality property. As in [Mo2,6E.1], now (using $\left.\mathrm{AC}_{\mathbf{R}}^{\omega}\right)$ we see that if $x=\left(a_{0}, a_{1}, \ldots\right)=\bigcup_{n} u_{n}$, then $x \in A$. From the definition of $x$ we see by a straightforward calculation that $x$ is in $\Delta$.

REMARKS. (A) Notice that in the above proof, from the properties of a very good quasiscale we only need (a), (b), (d), (e) and just the conclusion (3) from (c), i.e. the semicontinuity condition (4) is not needed (cf. [Mo2, 6E.1], which needs only a semiscale as opposed to a scale).

(B) For each set $A \subseteq \mathbf{R}$ consider the following game: I plays $x \in \mathbf{R}$, II plays $y \in \mathbf{R}$ (one integer at a time); I wins iff $x \in A$ (so it does not matter what II does). A winning strategy for $\mathrm{I}$ in this game is basically nothing more than a member of $A$. It is the application of the idea of the Third Periodicity Theorem to this degenerate game that we use here.

(C) It can be easily seen that if $\left\{S_{i}\right\}$ is actually a very good scale, then the procedure we use agrees with the canonical procedure explained before the preceding lemma (in that notation observe that, for a very good scale $\left\{\leq_{i}\right\}$, $A_{0}^{\prime}=A_{0}, A_{1}^{\prime}=A_{1}$, etc.).

2.4. In view of the lemma in 2.3 the claim in 2.2 will be proved, and our proof will be complete, if we can show the following final

LEMMA. Let $\alpha>1$. If $A \subseteq \mathbf{R}$ is $\Sigma_{1}$ definable over $J_{\alpha}(\mathbf{R})$ with parameters $r, \mathbf{R}, r$ a real, then $A$ has a quasiscale $\left\{\leq_{i}\right\}$ which is also $\Sigma_{1}$ definable over $J_{\alpha}(\mathbf{R})$ with parameters $r, \mathbf{R}$.

Indeed, granting this lemma, to finish the proof of the claim take $A \neq \varnothing$ to be $\Sigma_{1}$ definable over $J_{\alpha}(\mathbf{R})$ with parameters $r, \mathbf{R}$ and let $\Delta$ be the pointclass of all first-order definable in $J_{\alpha}(\mathbf{R})$ with parameters $r, \mathbf{R}$ sets. Then by the two preceding lemmas, $A$ contains a real in $\Delta$, and we are done.

PROOF OF THE LEMMA. This is basically nothing more than the realization that the proof of Steel [St, 2.1], that assuming ZF $+\mathrm{DC}+\mathrm{AD}+V=L(\mathbf{R})$ the set $A$ as above carries a scale which is $\Sigma_{1}$ definable over $J_{\alpha}(\mathbf{R})$ with parameters $r, \mathbf{R}$, can be slightly modified to give in $\mathrm{ZF}+\mathrm{AD}+V=L(\mathbf{R})$ only a quasicale with the same 
definability estimate. We sketch below the main steps of Steel's argument, pointing out the needed modifications.

2.5. First we will review the usual techniques for transferring scales (Second Periodicity Theorem, [Mo2]) and observe that they apply (with one modification) to quasiscales as well. It will be convenient here (in view of the use of these methods that we will make later on) to generalize a little the concept of quasiscale, so that it is applicable to relations with real and ordinal arguments.

Let $\lambda$ be a fixed ordinal. Consider relations $P \subseteq \mathbf{R}^{n} \times \lambda^{m}$. A quasiscale on $P$ is a sequence of relations $\left\{\leq_{i}\right\}$ on $P$ which satisfies conditions (a), (b), (c) of 2.3, where the notion of limit for points in $\mathbf{R}^{n} \times \lambda^{m}$ is defined in the obvious way, taking the topo$\log y$ on $\lambda$ to be discrete. Explicitly this means that $\left(x_{i}^{1}, x_{i}^{2}, \ldots, x_{i}^{n}, \eta_{i}^{1}, \ldots, \eta_{i}^{m}\right) \rightarrow$ $\left(x^{1}, x^{2}, \ldots, x^{n}, \eta^{1}, \ldots, \eta^{m}\right)$ iff $\forall k \leq n\left(x_{i}^{k} \rightarrow x^{k}\right) \wedge \forall l \leq m\left(\eta_{i}^{l}\right.$ is eventually constant and equal to $\eta^{l}$ ).

We have now the following transfer results:

(1) Assume $P \subseteq \mathbf{R}^{n+1} \times \lambda^{m}$ carries a quasiscale $\left\{S_{i}\right\}$. Define a very good quasiscale $\left\{\leq_{i}^{*}\right\}$ from $\left\{\leq_{i}\right\}$ as in the proof of the lemma in 2.3 (appropriately modified to handle finite sequences of reals; the ordinals are carried through as parameters). Consider then the relation

$$
S\left(x_{1}, \ldots, x_{n}, \eta_{1}, \ldots, \eta_{m}\right) \Leftrightarrow \forall y P\left(x_{1}, \ldots, x_{n}, y, \eta_{1}, \ldots, \eta_{m}\right) .
$$

Then we can define a quasiscale on $S$ as follows, imitating [Mo2, 6C.3].

Let $\tau_{0}, \tau_{1}, \ldots$ enumerate all finite sequences from $\omega$, so that $\tau_{0}=\varnothing$ and if $\tau_{i}$ is a proper initial segment of $\tau_{j}$ then $i<j$. Then for $(\bar{x}, \bar{\eta}),\left(\overline{x^{\prime}}, \overline{\eta^{\prime}}\right) \in S \operatorname{let}(\bar{x}, \bar{\eta}) \leq{ }^{i}\left(\overline{x^{\prime}}, \bar{\eta}^{\prime}\right)$ iff Player II has a winning strategy in the following game:

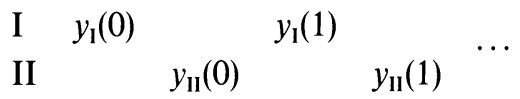

Let $y=\tau_{i}{ }^{n} y_{1}$ and $y^{\prime}=\tau_{i}{ }^{n} y_{\mathrm{II}}$; II wins iff $(\bar{x}, y, \bar{\eta}) \leq_{i}^{*}\left(\overline{x^{\prime}}, y^{\prime}, \overline{\eta^{\prime}}\right)$. That $\left\{\leq^{i}\right\}$ is a quasiscale on $S$ follows by exactly the same arguments as in [Mo2, C.3]. Note for further reference that the definition of $\leq^{i}$ involves only $\leq_{i}^{*}$ and thus only $\leq_{0}, \ldots, \leq_{i}$.

(2) Assume $P \subseteq \mathbf{R}^{n+1} \times \lambda^{m}$ carries a quasiscale $\left\{\leq_{i}\right\}$. Define again the very good quasiscale $\left\{\leq_{i}^{*}\right\}$ as before on $P$. Consider then the relation

$$
S(\bar{x}, \bar{\eta}) \Leftrightarrow \exists y P(\bar{x}, y, \bar{\eta}) .
$$

Then we can define a quasiscale on $S$ as follows.

For $(\bar{x}, \bar{\eta}),\left(\overline{x^{\prime}}, \overline{\eta^{\prime}}\right) \in S$, let $\left(\overline{x^{\prime}}, \overline{\eta^{\prime}}\right) \leq{ }^{i}(\bar{x}, \bar{\eta})$ iff Player II has a winning strategy in the following game:

$$
\begin{array}{llllll}
\text { I } & y(0) & & y(1) & & \\
\text { II } & & y^{\prime}(0) & & y^{\prime}(1) &
\end{array}
$$

II wins iff $\left(\overline{x^{\prime}}, y^{\prime}, \overline{\eta^{\prime}}\right) \in P \wedge\left[(\bar{x}, y, \bar{\eta}) \notin P \vee\left(\overline{x^{\prime}}, y^{\prime}, \overline{\eta^{\prime}}\right) \leq{ }_{i}^{*}(\bar{x}, y, \bar{\eta})\right]$.

REMARK. The motivation for this game, which one can call the "fake inf" game (in contrast with the "fake sup" game used in (1)), comes from the proof of the Norm Transfer Theorem in [Mo2, 6D.3]. In case $\left\{\leq_{i}\right\}$ is a scale, as opposed to a quasiscale, we can define a scale $\left\{\leq^{i}\right\}$ on $S$ by simply taking an infimum as in [Mo2, 6C.1], 
without playing a game. (The two definitions are the same in this case, as II wins the above game iff there is a $y^{\prime}$ such that $\left(\overline{x^{\prime}}, y^{\prime}, \overline{\eta^{\prime}}\right) \in P$ and for all $y$ with $(\bar{x}, y, \bar{\eta}) \in P$ we have $\left.\left(\overline{x^{\prime}}, y^{\prime}, \bar{\eta}^{\prime}\right) \leq{ }_{i}^{*}(\bar{x}, y, \bar{\eta})\right)$. Thus although the "fake inf" game is not needed if we have DC, thus also actual scales, it seems to be necessary in the absence of DC, and thus a symmetry between "fake sup" and "fake inf" is established. Note that we have also used a "fake inf" game in the lemma of 2.3.

By using the arguments in [Mo2,6D.3 and 6E.1] we can now verify easily that $\left\{\leq^{i}\right\}$ is a quasiscale on $S$. Again the definition of $\leq^{i}$ involves only $\leq_{0}, \ldots, \leq_{i}$.

(3) Finally assume $P \subseteq \mathbf{R}^{n} \times \lambda^{m+1}$ carries a quasiscale $\left\{\leq_{i}\right\}$. Let this time

$$
S(\bar{x}, \bar{\eta}) \Leftrightarrow \exists \xi P(\bar{x}, \bar{\eta}, \xi) \text {. }
$$

Then $S$ carries a quasiscale $\left\{\leq^{i}\right\}$ defined as follows:

$$
\begin{aligned}
(\bar{x}, \bar{\eta}) \leq{ }^{i}\left(\overline{x^{\prime}}, \overline{\eta^{\prime}}\right) \Leftrightarrow & \text { the least } \xi<\lambda \text { such that } P(\bar{x}, \bar{\eta}, \xi) \text { is } \\
& \text { strictly less than the least } \xi^{\prime} \text { with } P\left(\overline{x^{\prime}}, \overline{\eta^{\prime}}, \xi^{\prime}\right) \\
& \text { or else they are equal and }(\bar{x}, \bar{\eta}, \xi) \leq_{i}\left(\overline{x^{\prime}}, \overline{\eta^{\prime}}, \xi\right) .
\end{aligned}
$$

It is trivial to verify that this is indeed a quasiscale on $S$. The definition of $\leq{ }^{i}$ here involves only $\leq_{i}$.

For further use let us refer to the method in (1) for putting quasiscales on $\forall y P$ as the "fake sup" method, to that in (2) for putting quasiscales on $\exists y P$ as the "fake inf" method, and finally to the one in (3) for $\exists \xi P$ as the "min" method.

2.6. Let us say now that a set $P \subseteq \mathbf{R}$ has a closed game representation if there is a relation $T \subseteq\left(\omega^{<\omega}\right)^{<\omega} \times \lambda^{<\omega}$, for some ordinal $\lambda$, such that

$x \in P \Leftrightarrow \exists x_{0} \exists \eta_{0} \forall x_{1} \exists x_{2} \exists \eta_{1} \forall x_{3} \cdots \forall n\left(\left(x \uparrow n, x_{0} \uparrow n, x_{1} \uparrow n, \ldots, x_{2 n} \uparrow n\right),\left(\eta_{0}, \ldots, \eta_{n}\right)\right) \in T$.

The expression on the right means that player I has a winning quasistrategy in the following closed game:

$$
\begin{array}{llll}
\text { I } & x_{0}, \eta_{0} & & x_{2}, \eta_{1}
\end{array}
$$

$x_{i} \in \mathbf{R}, \eta_{i}<\lambda$; I wins iff

$$
\forall n\left(\left(x \uparrow n, x_{0} \uparrow n, \ldots, x_{2 n} \uparrow n\right),\left(\eta_{0}, \ldots, \eta_{n}\right)\right) \in T .
$$

Since we work in a context without choice (even without DC) we interpret this string as saying that I has a winning "quasistrategy" instead of "strategy". Explicitly a winning quasistrategy for $\mathrm{I}$ is a set $\mathscr{S}$ of finite sequences of the form $\left(x_{0}, \eta_{0}, x_{1}, \ldots, x_{2 n-2}, \eta_{n-1}, x_{2 n-1}\right)$ (for $n=0$ this is the empty sequence $\varnothing$ ) such that

(0) $\varnothing \in \mathscr{S}$;

(1) if $\left(x_{0}, \eta_{0}, x_{1}, \ldots, x_{2 n-2}, \eta_{n-1}, x_{2 n-1}\right) \in \mathscr{S}$ then there exist $x_{2 n}, \eta_{n}$ such that for all $x_{2 n+1}$,

$$
\left(x_{0}, \eta_{0}, x_{1}, \ldots, x_{2 n-2}, \eta_{n-1}, x_{2 n-1}, x_{2 n}, \eta_{n}, x_{2 n+1}\right) \in \mathscr{S}
$$

(2) if $\left(x_{0}, \eta_{0}, x_{1}, \ldots, x_{2 n}, \eta_{n}, x_{2 n+1}\right) \in \mathscr{S}$, then

$$
\left(\left(x \uparrow n, x_{0} \uparrow n, x_{1} \uparrow n, \ldots, x_{2 n} \uparrow n\right),\left(\eta_{0}, \ldots, \eta_{n}\right)\right) \in T \text {. }
$$

We can equivalently define this in terms of an inductive definition. This description will be useful later on in order to avoid some apparent uses of choice. 
Define the following monotone operator on the set of all sequences of the form $s=\left(x_{0}, \eta_{0}, x_{1}, \ldots, x_{2 n-2}, \eta_{n-1}, x_{2 n-1}\right)$ (including the empty one $\left.\varnothing\right)$ :

$s \in \Phi(X) \Leftrightarrow \forall z, \eta \exists y\left(s^{\cap}(z, \eta, y) \in X\right)$

$\vee\left[s \neq \varnothing \wedge\left(\left(x\left\lceil n-1, x_{0}\left\lceil n-1, \ldots, x_{2 n-2}\lceil n-1),\left(\eta_{0}, \ldots, \eta_{n-1}\right)\right) \notin T\right]\right.\right.\right.$.

Let $\Phi^{0}=\varnothing, \Phi^{\xi}=\Phi\left(\bigcup_{\theta<\xi} \Phi^{\theta}\right)$ and $\Phi^{\infty}=\bigcup_{\xi} \Phi^{\xi}=$ the least fixed point of $\Phi$. Then we claim that

$$
x \in P \Leftrightarrow \varnothing \notin \Phi^{\infty} .
$$

Indeed, if $\varnothing \notin \Phi^{\infty}$, then $\left\{s: s \notin \Phi^{\infty}\right\}$ is a winning quasistrategy for I. If $\varnothing \in \Phi^{\infty}$ but $x \in P$, let $\mathscr{S}$ be a quasistrategy for I demonstrating that $x \in P$. For each $s \in \Phi^{\infty}$ let $|s|=$ least $\xi$ such that $s \in \Phi^{\xi}$. Define then the following set of finite nonempty descending chains of ordinals:

$$
\begin{aligned}
\left(\xi_{0}, \xi_{1}, \ldots, \xi_{n}\right) \in C \Leftrightarrow \xi_{0}>\xi_{1}>\cdots>\xi_{n} \\
\wedge \exists x_{0}, \eta_{0}, x_{1}, \ldots, x_{2 n}, \eta_{n}, x_{2 n+1}\left\{\left(x_{0}, \eta_{0}, x_{1}, \ldots, x_{2 n}, \eta_{n}, x_{2 n+1}\right) \in \mathscr{S}\right. \\
\quad \wedge \forall i \leq n\left[\left(x_{0}, \eta_{0}, x_{1}, \ldots, x_{2 i}, \eta_{i}, x_{2 i+1}\right) \in \Phi^{\infty}\right. \\
\left.\left.\quad \wedge\left|\left(x_{0}, \eta_{0}, x_{1}, \ldots, x_{2 i}, \eta_{i}, x_{2 i+1}\right)\right|=\xi_{i}\right]\right\} .
\end{aligned}
$$

Clearly, $C \neq \varnothing$, and if $\left(\xi_{0}, \xi_{1}, \ldots, \xi_{n}\right) \in C$ there is $\xi_{n+1}$ with $\left(\xi_{0}, \xi_{1}, \ldots, \xi_{n}, \xi_{n+1}\right) \in C$, so we have an infinite descending chain of ordinals, a contradiction.

By combining now the three methods described earlier one can put a quasiscale on the set $P$ above as follows, copying the method of Moschovakis [Mo1].

Let, for each $k \geq 0$,

$$
\begin{aligned}
& P_{k}\left(x ; x_{0}, \eta_{0}, \ldots, x_{2 k-2}, \eta_{k-1}, x_{2 k-1}\right) \\
& \quad \Leftrightarrow \exists x_{2 k} \exists \eta_{k} \forall x_{2 k+1} \cdots \forall n\left(\left(x \left\lceiln, x_{0}\left\lceil n, \ldots, x_{2 n}\lceil n),\left(\eta_{0}, \ldots, \eta_{n}\right)\right) \in T\right.\right.\right.
\end{aligned}
$$

be the set of winning positions for player I of length $k$. Let also

$$
\begin{aligned}
& P_{k}^{+}\left(x ; x_{0}, \eta_{0}, \ldots, x_{2 k-2}, \eta_{k-1}, x_{2 k-1}, x_{2 k}\right) \\
& \quad \Leftrightarrow \exists \eta_{k} \forall x_{2 k+1} \cdots \forall n\left(\left(x \left\lceiln, x_{0}\left\lceil n, \ldots, x_{2 n}\lceil n),\left(\eta_{0}, \ldots, \eta_{n}\right)\right) \in T,\right.\right.\right. \\
& P_{k}^{++}\left(x ; x_{0}, \eta_{0}, \ldots, x_{2 k-2}, \eta_{k-1}, x_{2 k-1}, x_{2 k}, \eta_{k}\right) \\
& \quad \Leftrightarrow \forall x_{2 k+1} \cdots \forall n\left(\left(x \left\lceiln, x_{0}\left\lceil n, \ldots, x_{2 n}\lceil n),\left(\eta_{0}, \ldots, \eta_{n}\right)\right) \in T\right.\right.\right.
\end{aligned}
$$

be the set of appropriate incomplete winning positions for player I of length $k+1$. Then

$$
\begin{aligned}
P_{0}(x) & \Leftrightarrow P(x), \\
P_{k}\left(x ; x_{0}, \eta_{0}, \ldots, x_{2 k-1}\right) & \Leftrightarrow \exists x_{2 k} P_{k}^{+}\left(x ; x_{0}, \eta_{0}, \ldots, x_{2 k-1}, x_{2 k}\right), \\
P_{k}^{+}\left(x ; x_{0}, \eta_{0}, \ldots, x_{2 k}\right) & \Leftrightarrow \exists \eta_{k} P_{k}^{++}\left(x ; x_{0}, \eta_{0}, \ldots, x_{2 k}, \eta_{k}\right), \\
P_{k}^{++}\left(x ; x_{0}, \eta_{0}, \ldots, x_{2 k}, \eta_{k}\right) & \Leftrightarrow \forall x_{2 k+1} P_{k+1}\left(x ; x_{0}, \eta_{0}, \ldots, x_{2 k+1}\right),
\end{aligned}
$$

where the last equivalence follows immediately, without any use of choice, from the inductive analysis of winning a closed game, that we have explained before.

Define now quasiscales $\left\{\leq_{i}^{k}\right\},\left\{\leq_{i}^{+k}\right\},\left\{\leq_{i}^{++k}\right\}$ on $P_{k}, P_{k}^{+}, P_{k}^{++}$respectively as follows:

For $i<k, \leq_{i}^{k}, \leq_{i}^{+k}, \leq_{i}^{++k}$ are trivial, i.e. they hold for all pairs of elements in the corresponding sets. If $i \geq k$, then by induction on $i-k$ we proceed as follows:

(1) $\leq_{i}^{++k}$ is defined by the "fake sup" method from $\left\{\leq_{j}^{k+1}\right\}$. Since this needs only knowing $\leq_{0}^{k+1}, \ldots, \leq_{i}^{k+1}$, this is a legitimate definition. 
(2) $\leq_{i}^{+k}$ is defined by the "min" method from $\left\{\leq_{j}^{++k}\right\}$. This is legitimate, as it only requires knowing $\leq_{i}^{++k}$.

(3) $\leq_{i}^{k}$ is defined by the "fake inf" method from $\left\{\leq_{j}^{+k}\right\}$. This is again legitimate, as it requires knowing only $\leq_{0}^{+k}, \ldots, \leq_{i}^{+k}$.

Now it follows exactly as in Moschovakis [Mo1] that $\left\{\leq_{i}^{0}\right\}$ is a quasiscale on $P$.

2.7. Assume now $A \subseteq \mathbf{R}$ is $\Sigma_{1}$ definable over some $J_{\alpha}(\mathbf{R}), \alpha>1$, with parameters $r, \mathbf{R}$. For notational simplicity we consider only the case where $\alpha$ is limit-after all, the particular $\alpha$ we apply this lemma to is limit anyway. Also we drop the parameters $r, \mathbf{R}$, as their omission is not likely to cause any confusion. Thus for some $\Sigma_{1}$ formula $\varphi$

$$
x \in A \Leftrightarrow J_{\alpha}(\mathbf{R}) \vDash \varphi[x] \Leftrightarrow \exists \beta<\alpha\left(J_{\beta}(\mathbf{R}) \vDash \varphi[x]\right) .
$$

Put, for $\beta<\alpha$,

$$
x \in A_{\beta} \Leftrightarrow J_{\beta}(\mathbf{R}) \models \varphi[x] .
$$

Then $A=\bigcup_{\beta<\alpha} A_{\beta}$. Assume that we can put a quasiscale $\left\{\leq_{i}^{\beta}\right\}$ on each $A_{\beta}$ so that the map $(\beta, i) \mapsto \leq_{i}^{\beta}$ is $\Sigma_{1}$ over $J_{\alpha}(\mathbf{R})$. Then by the "min" method of 2.5 we can put a quasiscale on $A$, and it is easy to check that it is $\Sigma_{1}$ definable over $J_{\alpha}(\mathbf{R})$.

The quasiscale $\left\{\leq_{i}^{\beta}\right\}$ is constructed as in the proof of Steel [St, 2.1]. The key fact is that every $A_{\beta}$ admits a closed game representation (with ordinals $<\omega \beta$; recall that the ordinals in $J_{\beta}(\mathbf{R})$ are exactly those $<\omega \beta$ ). Steel's proof of this fact does not use DC-it is just a theorem of ZF. Moreover the corresponding set of sequences $T_{\beta}$ defining the closed game associated with $A_{\beta}$ is simple enough, so that if $\left\{\leq_{i}^{\beta}\right\}$ is the quasiscale on $A_{\beta}$ constructed by the Moschovakis procedure in 2.6, then $(\beta, i) \mapsto\left\{\leq_{i}^{\beta}\right\}$ is $\Sigma_{1}$ over $J_{\alpha}(\mathbf{R})$ (see again [St, 2.1]), and our proof is complete.

§3. The independence of $\mathrm{DC}$ and $\mathrm{AC}^{\omega}$ from $\mathrm{AD}$. It follows from $\S 2$ that

$$
\operatorname{Con}(\mathrm{ZF}+\mathrm{AD}) \Rightarrow \operatorname{Con}(\mathrm{ZF}+\mathrm{AD}+\mathrm{DC}) \text {. }
$$

The following result establishes the full independence of $\mathrm{DC}$ and $\mathrm{AC}^{\omega}$ from $\mathrm{ZF}+\mathrm{AD}$.

TheOrem (WoOdIN). Assume $\mathrm{ZF}+\mathrm{AD}+V=L(\mathbf{R})$. Then there is a symmetric generic extension of $V$ in which $\mathrm{AD}$ holds but $\mathrm{AC}^{\omega}$ fails. In particular,

$$
\operatorname{Con}(\mathrm{ZF}+\mathrm{AD}) \Rightarrow \operatorname{Con}\left(\mathrm{ZF}+\mathrm{AD}+\neg \mathrm{AC}^{\omega}\right) \text {. }
$$

Proof. By $\$ 2$ we have also $\mathrm{ZF}+\mathrm{AD}+\mathrm{DC}+V=L(\mathbf{R})$. Let $G$ be a generic enumeration of the set of reals in length $\omega_{1}$. We use of course as forcing conditions countable wellordered sequences of reals. Hence $V[G]=L(\mathbf{R})[G]=L[G](G$ is basically a subset of $\omega_{1}^{V[G]}$ ) and HOD is the same computed in $L(G]$ or $L(\mathbf{R})$ (also $\left.L(\mathbf{R})=L(\mathbf{R})^{L[G]}\right)$. Hence by Vopěnka's theorem ([J, p. 293]) $G$ is generic over HOD for some notion of forcing $\mathbf{P}$ which in $L[G]$ has cardinality $\leq \omega_{3}=\left(\Theta^{+}\right)^{V}$, where $\Theta=\sup \{\xi: \xi$ is a surjective image of $\mathbf{R}\}$.

Choose in $V(=L(\mathbf{R}))$ a regular cardinal $\lambda \gg \Theta$. Thus the cardinals above $\lambda$ are the same computed in HOD, $L(\mathbf{R})$ or $L[G]$. For each $\alpha>\lambda$ let $\mathbf{Q}_{\alpha}$ denote the forcing conditions defined in HOD for collapsing $\alpha$ to $\lambda$ via $<\lambda$-closed forcing, i.e., $\mathbf{Q}_{\alpha}=\left(\alpha^{<\lambda}\right)^{\mathrm{HOD}}$. 
Suppose $g$ is generic over $L[G]$ for $\mathbf{Q}_{\alpha}$. Then $G \times g$ is generic over HOD for $\mathbf{P} \times \mathbf{Q}_{\alpha}$, and $\operatorname{HOD}[G][g]=\operatorname{HOD}[g][G]$, while clearly $\lambda$ is not collapsed in $\operatorname{HOD}[g][G]$, so $\lambda$ is not collapsed and remains regular in $L[G, g]$. Further we see that $L(\mathbf{R})[g]$ contains no new sets of reals: If $A \subseteq \mathbf{R}, A \in L(\mathbf{R})[g]$, we will show that $A \in L(\mathbf{R})$. The generic object $g$ is canonically an onto function $f: \lambda \rightarrow \alpha$ such that $f \uparrow \beta \in \mathbf{Q}_{\alpha} \in \mathrm{HOD}, \forall \beta<\lambda$. Consider the following prewellordering on $\lambda$ :

$$
\gamma \preccurlyeq \gamma^{\prime} \Leftrightarrow f(\gamma) \leq f\left(\gamma^{\prime}\right) \text {. }
$$

Clearly $f$ is equiconstructible with $\preccurlyeq$. Now we have $L[G, \preccurlyeq] \vDash A \in L(\mathbf{R})[\preccurlyeq]$, and since $\lambda$ is regular in $L[G, \preccurlyeq]$ we have by a standard Skolem-Löwenheim argument that, for some $\xi, \beta<\lambda$,

$$
L_{\xi}[G, \preccurlyeq \uparrow \beta] \vDash A \in L(\mathbf{R})[\preccurlyeq \uparrow \beta] .
$$

But $\preccurlyeq \uparrow \beta \in L[f \uparrow \beta]$, so clearly $A \in L_{\xi}(\mathbf{R})[f \uparrow \beta] \subseteq L(\mathbf{R})$ and we are done.

Thus forcing with $\mathbf{Q}_{\alpha}$ over $L(\mathbf{R})$ does not collapse $\lambda$ or add new sets of reals. In particular AD holds in this generic extension.

For each $k \in \omega$ let now $\mathbf{Q}_{k}=\mathbf{Q}_{\lambda+k}$. Let $\mathbf{Q}=\mathbf{Q}_{1} \times \cdots \times \mathbf{Q}_{k} \times \cdots$ computed in HOD; thus $\mathbf{Q} \cong \mathbf{Q}_{\lambda+\omega}$. Suppose $G_{\mathbf{Q}}$ is generic for $\mathbf{Q}$ over $L(\mathbf{R})$. Then $L(\mathbf{R})\left[G_{\mathbf{Q}}\right] \vDash$ AD. Also $G_{\mathbf{Q}}=G_{\mathbf{Q}_{1}} \times \cdots \times G_{\mathbf{Q}_{k}} \times \cdots$, where each $G_{\mathbf{Q}_{k}}$ is generic over $L(\mathbf{R})$ for $\mathbf{Q}_{k}$ and canonically an onto function $f_{k}: \lambda \rightarrow \lambda^{+k}$, whose initial segments are in HOD.

By standard symmetry arguments and the homogeneity of $\mathbf{Q}$ we can find an inner model $L(\mathbf{R}) \varsubsetneqq \mathbf{N} \varsubsetneqq L(\mathbf{R})\left[G_{\mathbf{Q}}\right]$, such that $\mathbf{N} \models \mathrm{ZF}, f_{k} \in \mathbf{N}$ for each $k$ but $\lambda^{+\omega}$ is not collapsed in $\mathbf{N}$. Since $f_{k}$ collapses $\lambda^{+k}$ to $\lambda$ it follows that $\lambda^{+\omega}$ becomes $\lambda^{+}$in $\mathbf{N}$. Thus $\mathrm{AC}^{\omega}$ fails in $\mathbf{N}$ and because power( $(\mathbf{R}) \cap \mathbf{N}=$ power $(\mathbf{R}) \cap L(\mathbf{R}), \mathbf{N} \vDash \mathrm{AD}$. (To find such an $\mathbf{N}$ let, in $L(\mathbf{R})\left[G_{\mathbf{Q}}\right]$,

$$
\begin{aligned}
\mathscr{F}=\left\{f_{k}^{*}: \lambda \rightarrow \lambda^{+k} \mid k \in \omega, f_{k}^{*} \uparrow \beta \in \mathbf{Q}_{k} \text { for all } \beta<\lambda,\right. \\
\text { and } \left.f_{k}^{*}(\xi)=f_{k}(\xi), \text { for all sufficiently larger } \xi<\lambda\right\} .
\end{aligned}
$$

Let $A=\mathscr{F} \cup \mathbf{R}$ and $\mathbf{N}=\operatorname{HOD}_{A}^{L(\mathbf{R})\left[G_{\mathbf{Q}}\right]}$, i.e. all sets hereditarily ordinal definable in $L(\mathbf{R})\left[G_{\mathbf{Q}}\right]$ with parameters in $\mathscr{F} \cup\{\mathscr{F}\} \cup \mathbf{R}$.

\$4. On the strength of $\mathbf{Z F}+\mathbf{A D}+\neg \mathbf{D C}_{\mathbf{R}}$. The following consequence of the main result was noted by $\mathrm{H}$. Woodin and the author.

Theorem. Assume $\mathrm{ZF}+\mathrm{AD}+\neg \mathrm{DC}_{\mathbf{R}}$. Then $\mathbf{R}^{\#}$ exists. In particular, we have $\operatorname{Con}(\mathrm{ZF}+\mathrm{AD}+\mathrm{DC})$.

Proof. Assume $\mathrm{ZF}+\mathrm{AD}+\neg \mathrm{DC}_{\mathbf{R}}$. Then $L(\mathbf{R}) \models \mathrm{ZF}+\mathrm{AD}$; thus by $\S 2$, $L(\mathbf{R})=\mathrm{ZF}+\mathrm{AD}+\mathrm{DC}$. So there must be some $A \subseteq \mathbf{R}, A \notin L(\mathbf{R})$. By Wadge's lemma (see [Mo2]) we must have then that there is some $A \notin L(\mathbf{R})$ such that every $B \in L(\mathbf{R}), B \subseteq \mathbf{R}$ is a continuous preimage of $A$. As an obvious corollary, $\Theta>\Theta^{L(\mathbf{R})}$. By standard results from AD (see for example [Mo2], $[\mathrm{K}]$ ) it follows that $\Theta>\left(\Theta^{+}\right)^{L(\mathbf{R})}$ and there is a measurable cardinal $\kappa>\left(\Theta^{+}\right)^{L(\mathbf{R})}$. But then for all $X \subseteq\left(\Theta^{+}\right)^{L(\mathbf{R})}, X^{\#}$ exists.

Now as in $\S 3$, if $G$ is a generic over $L(\mathbf{R})$ enumeration of the reals in length $\omega_{1}$, we see that $L(\mathbf{R})[G]=L[G], L(\mathbf{R})=L(\mathbf{R})^{L[G]}$ and $G$ is basically a subset of $\omega_{1}^{L[G]}$, so that by Vopěnka's theorem $\operatorname{HOD}^{L[G]}=\operatorname{HOD}^{L(\mathbf{R})}=L[P]$, where $P \subseteq \omega_{3}^{L[G]}=$ $\left(\Theta^{+}\right)^{L(\mathbf{R})}$, and $L[G]$ is a generic extension of $L[P]$ (in $[K W]$ it is actually shown 
that $\operatorname{HOD}^{L(\mathbf{R})}=L[Q]$, for $\left.Q \subseteq \Theta^{L(\mathbf{R})}\right)$. So since $P^{\#}$ exists, we conclude that $\mathbf{R}^{\#}$ exists as well.

(Note that the above argument shows that $\mathrm{ZF}+\mathrm{AD}+\exists A \subseteq \mathbf{R}(A \notin L(\mathbf{R})) \Rightarrow \mathbf{R}$ \# exists. If we add $\mathrm{DC}_{\mathbf{R}}$ to the hypotheses this result was proved in [St-VW]).

§5. In the realm of projective sets. Consider now the language of second order number theory or analysis (see for example [Sh, 8.5]), together with the usual basic axioms including induction and extensionality. The Comprehension Axiom Schema (CA-Schema) consists of all axioms of the form

$$
\exists x \forall n(n \in x \Leftrightarrow \varphi(n)) .
$$

Here $x$ varies over subsets of $\omega, n$ over $\omega$, and $\varphi$ is a formula in analysis containing possibly parameters. We use these same conventions below as well.

The Dependent Choices Schema (DC-Schema) consists of the axioms

$$
\forall x \exists y \varphi(x, y) \Rightarrow \exists y \forall n \varphi\left((y)_{n},(y)_{n+1}\right),
$$

where, for $y \subseteq \omega,(y)_{n}=\left\{m: 2^{n} \cdot 3^{m} \in y\right\}$.

Finally the Projective Determinacy Schema (PD-Schema) consists of the axioms

$$
\{x: \varphi(x)\} \text { is determined. }
$$

The theory of projective sets from projective determinacy (see [Mo2]) can be developed within analysis from CA-Schema + DC-Schema + PD-Schema.

Let $T_{0}$ be the theory in analysis containing beyond the basic axioms also the comprehension axioms for arithmetical formulas only (actually much less is needed below). Then notice the following obvious fact:

In $T_{0}$,

$$
\text { PD-Schema } \Rightarrow \text { CA-Schema }
$$

(given $\varphi(n)$, consider the game where I plays, $n, n_{0}, n_{1}, \ldots$, II plays, $i, i_{0}, i_{1}, \ldots$ and II wins iff $\varphi(n) \Leftrightarrow i=0)$.

From the work in $\S 2$, it now follows easily that, in $T_{0}$,

$$
\text { PD-Schema } \Rightarrow \text { DC-Schema. }
$$

Thus PD-Schema (added to $T_{0}$ ) by itself is sufficient for the development of the theory of projective sets in analysis. (With a little patience the interested reader can actually obtain finer level-by-level versions of these results in the projective hierarchy).

\section{REFERENCES}

[J] T. JeCH, Set theory, Academic Press, New York, 1980.

[K] A.S. KECHRIS, AD and projective ordinals, Cabal Seminar 76-77 (A.S. Kechris and Y. N. Moschovakis, editors), Lecture Notes in Mathematics, vol. 689, Springer-Verlag, Berlin, 1978, pp. 91-132.

[KMM] A. S. Kechris, D. A. Martin and Y. N. MoschovaKis (editors), Cabal Seminar 79-81, Lecture Notes in Mathematics, Vol. 1019, Springer-Verlag, Berlin, 1983.

[KW] A. S. KECHRIS and W. H. Woodin, The structure of $\Theta$ in $L(\mathbf{R})$ (to appear).

[Ma] D. A. MARTIN, The axiom of determinateness and reduction principles in the analytical hierarchy, Bulletin of the American Mathematical Society, vol. 74 (1968), pp. 687-689. 
[MMS] A. D. Martin, Y. N. Moschovakis and J. R. Steel, The extent of definable scales, Bulletin (New Series) of the American Mathematical Society, vol. 6 (1982), pp. 435-440.

[Mo1] Y. N. MoschovaKis, Scales on coinductive sets, [KMM], pp. 81-91.

[Mo2] - Descriptive set theory, North-Holland, Amsterdam, 1980.

[Mo3] - Determinacy and prewellorderings of the continuum, Mathematical logic and foundations of set theory (Y. Bar-Hillel, editor), North-Holland, Amsterdam, 1970, pp. 24-62.

[My] J. Mycielski, On the axiom of determinateness. I, II, Fundamental Mathematicae, vol. 53 (1963/64), pp. 205-224, and vol. 59 (1966), pp. 203-212.

[Sh] J. R. Shoenfield, Mathematical logic, Addison-Wesley, Reading, Massachusetts, 1967.

[So] R. M. SolovaY, The independence of DC from AD, Cabal Seminar 76-77 (A. S. Kechris and Y. N. Moschovakis, editors), Lecture Notes in Mathematics, vol. 689, Springer-Verlag, Berlin, 1978, pp. $171-184$.

[St] J. R. STEEL, Scales in L(R), [KMM], pp. 113-163.

[St-VW] J. R. STEEL and R. VAN WESEP, Two consequences of determinacy consistent with choice, Transactions of the American Mathematical Society, vol. 272 (1982), pp. 67-87.

CALIFORNIA INSTITUTE OF TECHNOLOGY

PASADENA, CALIFORNIA 91125 